\title{
Derivation of Mean Value Coordinates Using Interior Distance and Their Application on Mesh Deformation
}

\author{
Derivação de coordenadas do valor médio usando distância interior e sua aplicação em \\ deformação de malhas
}

\author{
Lis Custódio ${ }^{1 *}$, Sinesio Pesco²
}

\begin{abstract}
The deformation methods based on cage controls became a subject of considerable interest due its simplicity and intuitive results. In this technique, the model is enclosed within a simpler mesh (the cage) and its points are expressed as function of the cage elements. Then, by manipulating the cage, the respective deformation is obtained on the model in its interior.

In this direction, in the last years, extensions of barycentric coordinates, such as Mean Value coordinates, Positive Mean Value Coordinates, Harmonic coordinates and Green's coordinates, have been proposed to write the points of the model as a function of the cage elements.

The Mean Value coordinates, proposed by Floater in two dimensions and extended later to three dimensions by Ju et al. and also by Floater, stands out from the other coordinates because of their simple derivation. However the existence of negative coordinates in regions bounded by non-convex cage control results in a unexpected behavior of the deformation in some regions of the model.

In this work, we propose a modification in the derivation of Mean Value Coordinates proposed by Floater. Our derivation maintains the simplicity of the construction of the coordinates and eliminates the undesired behavior in the deformation by diminishing the negative influence of a control vertex on regions of the model not related to it. We also compare the deformation generated with our coordinates and the deformations obtained with the original Mean Value coordinates and Harmonic coordinates.
\end{abstract}

Keywords: Mesh deformation - Barycentric coordinates - Mean value coordinates - Interior distance

Resumo: Os métodos de deformação baseados em poliedros de controle tornaram-se um assunto de considerável interesse devido a sua simplicidade e resultados intuitivos. Nesta técnica, o modelo é envolto em uma malha mais simples (o poliedro de controle) e seus pontos são escritos em função dos elementos desse poliedro. Dessa forma, manipulando o poliedro de controle, a respectiva deformação é obtida no modelo em seu interior.

Nessa direção, nos últimos anos, extensões de coordenadas baricêntricas, como as coordenadas de Valor Médio, coordenadas de Valor Médio Positivas, coordenadas Harmônicas e coordenadas de Green, têm sido propostas para escrever os pontos do modelo em função dos elementos do poliedro de controle.

As Coordenadas do Valor Médio, propostas por Floater em duas dimensões e estendidas posteriormente para três dimensões por Ju et al. e também por Floater, destacam-se por sua simples derivação. No entanto, a existência de coordenadas negativas em regiões delimitadas por poliedros de controle não-convexos resulta em um comportamento inesperado da deformação em algumas regiões do modelo.

Neste trabalho, propomos uma modificação na derivação de coordenadas de Valor Médio proposta por Floater. Nossa derivação mantém a simplicidade da construção das coordenadas e elimina o comportamento indesejado na deformação diminuindo a influência negativa de um vértice de controle em regiões do modelo não relacionadas a ele. Também comparamos a deformação gerada com as nossas coordenadas e as deformações obtidas com as coordenadas do Valor Médio originais e as coordenadas Harmônicas.

Palavras-Chave: Deformação de malhas - Coordenadas baricêntricas - Coordenadas do valor médio Distância interior

${ }^{1}$ Department of computational modeling, Polytechnic Institute, Rio de Janeiro State University, Rio de Janeiro, Rio de Janeiro, Brazil

${ }^{2}$ Department of Mathematics, Pontifical Catholic University of Rio de Janeiro, Rio de Janeiro, Rio de Janeiro, Brazil

*Lis Custódio: lis.custodio@uerj.br

DOI: http://dx.doi.org/10.22456/2175-2745.76189 • Received: 18/09/2017 • Accepted: 19/04/2018

CC BY-NC-ND 4.0 - This work is licensed under a Creative Commons Attribution-NonCommercial-NoDerivatives 4.0 International License. 
Deformations methods based on cage control stand out from the others methods due their fast and intuitive interaction. In these techniques the model is enclosed in a cage with a considerably smaller number of vertices (compared with the model), then the vertices of the model are defined as a function of the vertices of the cage (the control vertices) ([1], [2], [3]). Thus, the subsequents deformations of the cage control uniquely define the deformations of the model in its interior.

Some deformation techniques use extensions of the barycentric coordinates to achieve the reconstruction of the model in function of the cage control. The barycentric coordinates $\lambda_{i}$ of a point $v_{0}$ in the interior of a region $P$ (polygons in $\mathbb{R}^{2}$ and polyhedras in $\mathbb{R}^{3}$ ) satisfy the following criteria:

$$
\begin{aligned}
& \lambda_{i} \geq 0, \text { positivity. } \\
& \sum_{i=1}^{n} \lambda_{i}=1, \text { partition of unity. } \\
& \sum_{i=1}^{n} \lambda_{i} v_{i}=v_{0}, \text { linear precision. }
\end{aligned}
$$

Where, $n$ is the number of vertices and $v_{i}$ are the coordinates of the vertices in the boundary of $P$.

For simplexes (triangles in $\mathbb{R}^{2}$ and tetrahedra in $\mathbb{R}^{3}$ ), the system of equations (1) has a single solution. However, if we take a polygon with more than three vertices, the system of equations (1) becomes consistent and indeterminate, and there are infinite solutions that describe the point $v_{0}$ in terms of vertices $v_{i}, i=1, \cdots, n$. The same happen in $\mathbb{R}^{3}$ for a polyhedra with more than four vertices.

Consider a region $P$ with boundary $\partial P$ defined by a polygon with vertices $v_{1}, v_{2}, \ldots, v_{n}$ arranged in counter-clockwise order and a point $v_{0}$ in the interior of this region (see Figure 1(a)). One can interpret the coordinate $\lambda_{i}$ of point the $v_{0}$ in related to the vertex $v_{i}$ as the influence of the vertex $v_{i}$ on the point $v_{0}$. When attempting to extend the barycentric coordinates to non-simplex polyhedra, we are actually attempting to smoothly propagate the influence of a vertices on the boundary $\partial P$ of region to its interior $\operatorname{int}(P)$. We define the function $f$ to be the identity function on $\partial P$, and we seek a smooth function $u$ defined in $\operatorname{int}(P)$ such that $u$ is equal to $f$ for every point on $\partial P$. This smoothness is obtained once we require that $u$ be a harmonic function. In other words, we seek to obtain a function $u$ defined in $P$ such that $u$ is a solution to the Dirichlet problem inside region $P$, with the identity function as the boundary condition:

$$
\nabla^{2} u(v)=0, \quad v \in \operatorname{int}(P) \quad \text { and } \quad u(v)=f(v), \quad v \in \partial P .
$$

In 2003, Floater [4] proposed a coordinate system based on the mean value theorem [2] that yields smooth coordinates in regions bounded by closed polygons. In 2005, Ju et al. [5] and Floater et al. [6] extended the coordinate system obtained by Floater to the three-dimensional space. The Mean value coordinates (MVC) are distinct from other extensions of barycentric coordinates because of their simple derivation, with closed formula to derivate the coordinates in two and three dimension, and local influence of control points, which result in an intuitive deformation process. However, for nonconvex cage control, the presence of negative coordinates results in an undesired behavior of the deformation (problem illustrated in the Section 2). In this work, to eliminate these undesired behavior, we propose a new approach in the derivation of the Mean Value Coordinates, in which a control vertex do not has a significant influence on regions not related to it.

This work is organized as follows. In Section 1, we highlight the main characteristics of the deformation methods that use barycentric coordinates proposed in the last years. In Section 2, we present the derivation of MVC proposed by Floater, and point out the causes and consequences of negative coordinates. In Section 3, we propose a modification on the derivation proposed by Floater, where the Euclidean distance is replaced by the interior distance, which better respect the model geometry. In Section 4, we present the deformations obtained with our coordinates and compare them with the deformations obtained using MVC and harmonic coordinates.

\section{Related work}

Although barycentric coordinates have several applications in computer graphics (some of them can bee seen in the works proposed by Li et al. [7], Rustamov [8] and Farbman et al. [9]), we opt to restrict the related works to their application in mesh deformation.

In 2005, Ju et al [5] and Floater et al. [6] extended to three dimensions the Mean Value Coordinates proposed by Floater [4] in 2003. The derivation, based on the mean value theorem for harmonic functions, has a closed formula and results in a intuitive deformation process. However, the non-negativity of the coordinates is guaranteed only on regions bounded by convex cage control.

In 2007, Joshi et al. [10] proposed the Harmonic Coordinates (HC), a generalization of barycentric coordinates derived by the numerical solution of the Laplace's equation inside the cage control, which yielded strictly positive coordinates. The cage control is filled by a regular grid, the solution of the Laplace's equation is obtained on its cells and then it is interpolated to the vertices of the model. The necessity of the domain discretization makes the derivation of the Harmonic Coordinates a slow process, in which the linear precision is guaranteed only on the grid cells.

Also in 2007, Lipman et al. [11] proposed the construction of strictly positive coordinates derived from the mean value theorem, in which the visibility of a point within the control polyhedron is considered, i.e., points within the cage control are written only in terms of those (control) points that are visible to it. In this way every interior point has a convex cage control, which avoids the negative coordinates presented in the method proposed by Ju et al. [5]. The visibility criterion makes neighbors regions on the model be controlled by different cage controls, which compromise the smoothness of the coordinates. 
In 2008, Lipman et al. [12] proposed coordinates based on the Green's third identity, which describe points in the interior of the cage control in terms of its vertices and faces. Green's Coordinates (GC) yield a conformal mapping in two dimensions and a quasi-conformal one in three dimensions. However, conformal mappings are restricted to rotations and scalings, and the global influence generated by the method in every control vertex results in a deformation process that is difficult to control.

In 2010, Manson and Schaefer [13] constructed extensions of barycentric coordinates based on least squares. These moving least squares coordinates, similarly to the MVC, yield negative coordinates for non-convex cage control. Moreover, there is no closed formula to derivate the coordinates in three dimensional space.

\section{The Mean Value Coordinates}

In this section we present the derivation of the Mean Value Coordinates proposed by Floater [4]. For the simplicity of the explanation we opt to present only the derivation in two dimension, since the derivation in three dimension is based on the same argument. For more details on the derivation and analysis of MVC in three dimensions, see Custodio [14].

Let $P$ be a region of the plane delimited by a convex polygon with vertices $v_{1}, v_{2}, \ldots, v_{n}$, arranged in counter-clockwise order, and $u$ be a harmonic function defined on its interior. Given a point $v_{0}$ inside $P$, we denote by $B\left(v_{0}, r\right)$ the circumference centered at $v_{0}$ with radius $r$ (see Figure 1(a)).

In his work, Floater [4] use the Mean Value Theorem to harmonic function to write the value of the function $u$ at the point $v_{0}$ as a function of the a linear function defined on the boundary of $P$.

Theorem 1 (Circumferential mean value theorem) Let $P \subset$ $\mathbb{R}^{2}$ be a convex region, $u$ be a harmonic function in $P$ and $B=B\left(v_{0}, r\right) \subset P$ with boundary $\Gamma$; then,

$$
u\left(v_{0}\right)=\frac{1}{2 \pi r} \int_{\Gamma} u(v) d s .
$$

Consider the triangle $T_{i}=\left[v_{0}, v_{i}, v_{i+1}\right]$ (see Figure 1) and let $\Gamma_{i}$ be the arc of $\Gamma$ contained in $T_{i}$. We can now rewrite equation (3) as

$$
u\left(v_{0}\right)=\frac{1}{2 \pi r} \sum_{i=1}^{n} \int_{\Gamma_{i}} u(v) d s .
$$

Lemma 1 If $f: \Gamma_{i} \rightarrow \mathbb{R}$ is a linear function and $\alpha_{i}$ is the signed angle between vertices $v_{i}$ and $v_{i+1}$, then

$$
\begin{gathered}
\int_{\Gamma_{i}} f(v) d s=r^{2} \tan \left(\frac{\alpha_{i}}{2}\right)\left(\frac{f\left(v_{i}\right)-}{\left\|v_{i}-v_{0}\right\|}+\frac{f\left(v_{i+1}\right)-f\left(v_{0}\right)}{\left\|v_{i+1}-v_{0}\right\|}\right) \\
+r \alpha_{i} f\left(v_{0}\right) .
\end{gathered}
$$

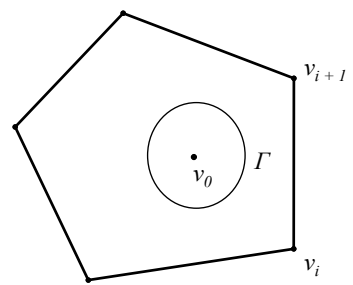

(a)

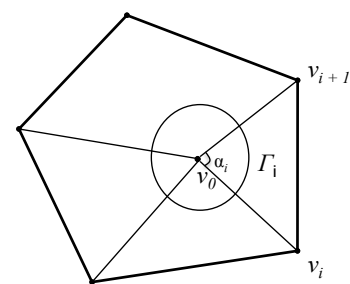

(b)
Figure 1. (a) Circumference $\Gamma$ centered at $v_{0}$ inside region $P$. (b) Triangulation of region $P$.

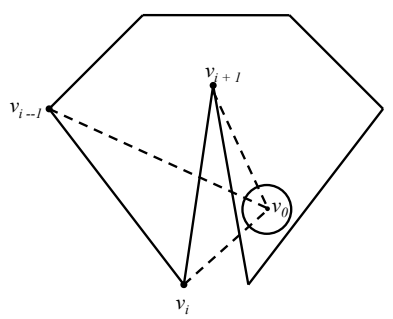

Figure 2. Case leading to negative coordinates.

The Lemma 1 allows us to rewrite the Equation (4):

$$
u\left(v_{0}\right)=\sum_{i=1}^{n} \frac{w_{i}}{\sum_{i=1}^{n} w_{i}} u\left(v_{i}\right), \quad w_{i}=\left(\frac{\tan \frac{\alpha_{i}}{2}+\tan \frac{\alpha_{i-1}}{2}}{\left\|v_{i}-v_{0}\right\|}\right)
$$

Denoting

$$
\lambda_{i}=\frac{w_{i}}{\sum_{i=1}^{n} w_{i}}
$$

yields

$$
u\left(v_{0}\right)=\sum_{i=1}^{n} \lambda_{i} u\left(v_{i}\right)
$$

Note that Equations (6), (7) and (8) imply, respectively, that the obtained coordinates are positive, their sum is equal to 1 and they have linear precision when representing a point in the interior of region $P$, i.e., $\lambda_{i}$ are the barycentric coordinates for $v_{0}$ relative to vertices $v_{i}$.

\subsection{The Negative Coordinates}

Consider now a region $P$ in the plane, bounded by a nonconvex polygon.

As showed in the Figure 2, the edge $\left[v_{i}, v_{i+1}\right]$ is projected on the circumference centered at $v_{0}$ with its reverse orientation; therefore, the coordinate $w_{i}$ of the vertex $v_{i}$ relative to the vertex $v_{0}$ will, be given by

$$
w_{i}=\left(\frac{\tan \left(\frac{-\alpha_{i}}{2}\right)+\tan \frac{\alpha_{i-1}}{2}}{\left\|v_{i}-v_{0}\right\|}\right) \text {, }
$$

where $\tan \left(\frac{-\alpha_{i}}{2}\right)<0$ and $\left|\tan \left(\frac{\alpha_{i+1}}{2}\right)\right|<\left|\tan \left(\frac{-\alpha_{i}}{2}\right)\right|$, which imply $w_{i}<0$. 


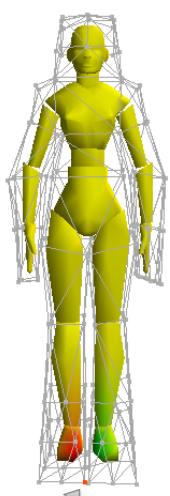

(a)

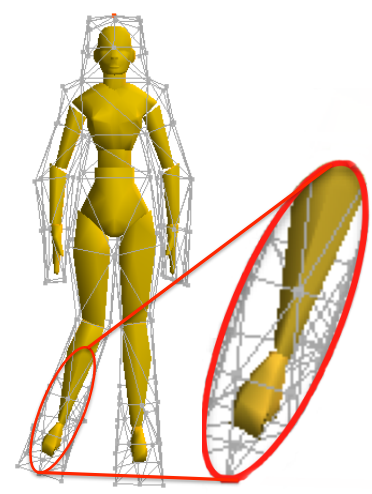

(b)
Figure 3. (a) Positive (red) and negative (green) influence regions of the orange-colored vertex. (b) Consequence of negative weights and use of Euclidean distance in MVC.

\subsection{Consequences of negative coordinates}

The consequence of the generation of negative coordinates is that in the deformation process some regions of the model present an undesired behave. In Figure 3(a), we highlight the regions where the control vertex in orange has the greatest influence. In red the region where this influence is positive and in green the region of negative influence. In the Figure 3(b) is possible to observe that the movement of the control points related to the model's left leg causes an undesired deformation in the right leg. The same effect can be observed on the left leg, caused by the negative influence from the control vertices of the right leg.

The problem caused by the negative coordinates is aggravated by the use the Euclidean distance during the derivation of the coordinates. When considering the Euclidean distance between control vertices and points of the model, the visibility of a point inside the cage control is not respected. Thus, the control vertices that are far from a point of the model, considering path within the cage control, are by the euclidian distance as close as the control vertices directly related to this point (see Figure 4). Therefore, a region of the model may suffer great influence from control vertices that are not directly related to it, that will be negative influence, as showed in Figure 3.

\section{Derivation of Mean Value Coordinates using Interior Distance}

As presented in Section 2.1, MVC do not satisfy the assumption of non-negativity for non-convex cage control. In the Figure 5 we present some examples of this negative influence.

To solve the problem of the great influence of the control vertices on regions not related to them we propose a modification on the derivation of the Mean Value Coordinates, we replace the use of the euclidian distance by the interior distance given by the length a path within the cage control (see Figure 6), and we call the resulting coordinates by Mean Value Coordinates with interior distance (MVC-ID).

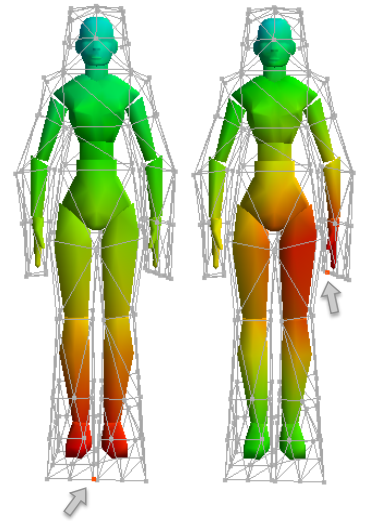

Figure 4. Propagation of Euclidean distance from orange-colored control vertices over the model.

We present a process of derivation that maintains the simplicity of the MVC derivation by Floater [4]. In our derivation it is not necessary to discretize the domain, and the smoothness of the resulting coordinates is not compromised, which are disadvantages of harmonic (Joshi et al. [10]) and positive MVC (Lipman et al. [11]), respectively.
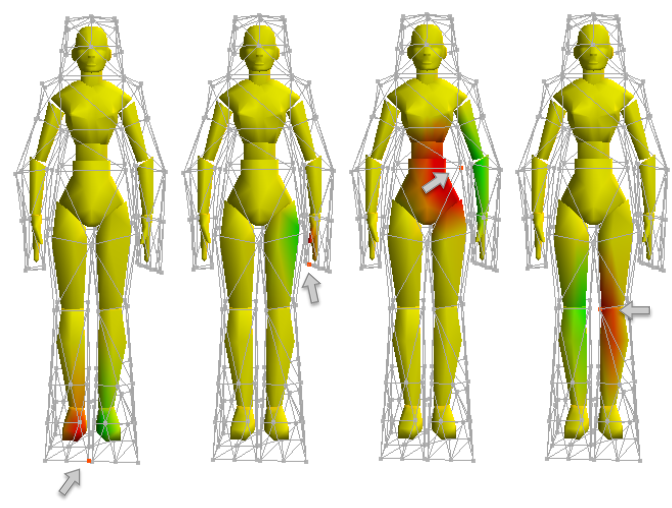

Figure 5. Influence regions of the orange-colored vertices. Positive influence varies from red to yellow, and negative influence varies from green to yellow.

\subsection{The Interior Distance}

Consider a distance $d$ measured over the mesh of the cage control and the distances $d_{i j}=\left(v_{i}, v_{j}\right)$ between the vertices in this mesh. Once we have a distance measure over the mesh of the cage control, we define another distance measure in its interior based on the article Interior Distance Using Barycentric Coordinates (Rustamov et al. [15]), in which extensions of barycentric coordinates are used to propagate distances measured on a mesh to its interior.

To this end, the control vertices $v_{i} \in \mathbb{R}^{3}$ are embedded in a higher dimensional space $\mathbb{R}^{m}$ (for some $m$ ) by performing a mapping $v_{i} \mapsto v_{i}{ }^{*} \in \mathbb{R}^{m}$ such that $\left\|v_{i}{ }^{*}-v_{j}{ }^{*}\right\|_{2}=d_{i j}$. Next, using barycentric coordinates, we extend this mapping to the interior of the mesh.

Given a point $p$ inside the cage control and its barycentric 
(a)

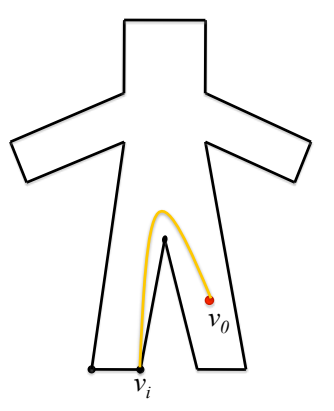

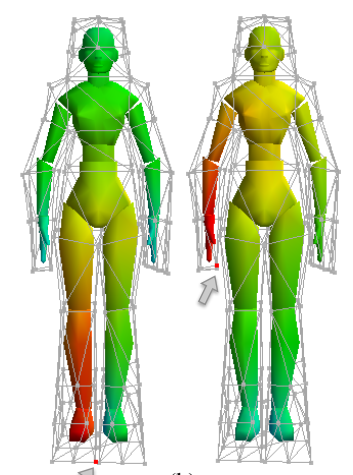

(b)
Figure 6. (a) Illustrative example of a path inside the control polygon. (b) Propagation of interior distance from control vertices, highlighted in orange, over the model.

coordinates relative to the control vertices $\lambda_{i}(p), i=1, \ldots, n$ (where $n$ is the number of control vertices), we have:

$$
p \mapsto p^{*}=\sum_{i} \lambda_{i}(p) v_{i}^{*}
$$

The distance between two points, $p$ and $q$, taken inside the cage control is given by the Euclidean distance of their images, $p^{*}$ and $q^{*}$ :

$$
\hat{d}(p, q)=\left\|p^{*}-q^{*}\right\|_{2}
$$

The interior distance is therefore given as a function of the barycentric coordinates of points $p$ and $q$ and of the images $v_{i}^{*}$ of the control vertices.

Taking $p^{*}=\sum_{i} \lambda_{i}(p) v_{i}^{*}$ e $q^{*}=\sum_{i} \lambda_{i}(q) v_{i}{ }^{*}$, from Equation (11) we have:

$$
\begin{aligned}
\hat{d}^{2}(p, d) & =\left\langle p^{*}-q^{*}, p^{*}-q^{*}\right\rangle \\
& =\left\langle\sum_{i}\left(\lambda_{i}(p)-\lambda_{i}(q)\right) v_{i}{ }^{*}, \sum_{j}\left(\lambda_{j}(p)-\lambda_{j}(q)\right) v_{j}{ }^{*}\right\rangle \\
& =\sum_{i, j}\left(\lambda_{i}(p)-\lambda_{i}(q)\right)\left(\lambda_{j}(p)-\lambda_{j}(q)\right)\left\langle v_{i}{ }^{*}, v_{j}{ }^{*}\right\rangle \\
& =(\vec{\lambda}(p)-\vec{\lambda}(q))^{T} A(\vec{\lambda}(p)-\vec{\lambda}(q)),
\end{aligned}
$$

where $\vec{\lambda}(p)=\left(\lambda_{1}(p), \lambda_{2}(p), \ldots, \lambda_{n}(p)\right)$ is the column vector of the weights of the control vertices related to the point $p$ and matrix $A$ is the Gram matrix of the vertices, where each element is denoted by $a_{i j}=\left\langle v_{i}{ }^{*}, v_{j}{ }^{*}\right\rangle_{\mathbb{R}^{m}}$. The interior distance of a control polyhedron vertex to a point in its interior is thus given by $\hat{d}\left(v_{i}, p\right)$.

In our implementation, we opt do not to use an explicit embedding. We computed the matrix A directly, using multidimensional scaling (MDS) ((Ju et al. [16]).

Let $D$ be the matrix with the square distances as measured over the cage control, with $D_{i j}=d^{2}\left(v_{i}, v_{j}\right)$ and $I$ being the identity matrix, then:

$$
J=I-\frac{1}{n} \overrightarrow{1}^{1} \overrightarrow{1}^{T},
$$

where $\overrightarrow{1}$ is a column vector of 1's. Thus, A can be written as:

$$
A=-\frac{1}{2} J D J
$$

For simplicity, we opt to define the distance between cage control vertices as the shortest path between them, taken over the edges of the mesh. In addition, to simplify the implementation, we used the original MVC to extend the embedding performed at the control polyhedron vertices into its interior, as suggested in the work of Rustamov et al. [15]. Despite the negative coordinates, the original MVC guarantee the linear precision in the reconstruction of points within the cage control.

\subsection{Properties of interior distance}

- Interpolation The distance $\hat{d}$ obtained inside the mesh interpolates the distance $d$ measured on the mesh.

- Metric: If the Gram matrix $A$ is a positive definite matrix, then the distance $\hat{d}$ is a metric.

- Same topology as Euclidean distance: If the Gram matrix $A$ is a positive definite matrix and the barycentric coordinates are continuous, then the distance $\hat{d}$ induces the same topology of isolines in the interior of the mesh as the Euclidean distance.

\subsection{The Use of Interior Distance in the Derivation of Mean Value Coordinates}

The nature of the method presented in the Section 3.1 to the computation of the interior distance makes it inherit the characteristic of the distance measured on the mesh. Thus, chose of distance as the shortest path (choose due its simple computation) as the distance to be propagate to the interior of the cage control will result in an interior distance unable to guarantee the linear precision of our coordinates and, consequently, there will be an error in the reconstruction of a given model point $p$ :

$$
p=\sum_{i=1}^{n} \gamma_{i} v_{i}+\varepsilon, \quad i=1 \cdots n .
$$

Where $n$ is the number of control vertices, $\gamma_{i}$ is the MVCID of the point $p$ related to the control vertex $v_{i}$ and $\varepsilon \in \mathbb{R}^{3}$ is a vector which coordinates are the errors obtained in the reconstruction of the point $p$ in the axis $x, y$ and $z$.

To solve the reconstruction problem and achieve the linear precision in our coordinates we introduce the following interactive method.

- For each point of the model, we write the reconstruction $\varepsilon$ (Equation 15) as function of the control points and its MVC-ID.

$$
\varepsilon=\sum_{i=1}^{n} \gamma_{i}^{\varepsilon} v_{i}+\varepsilon, \quad i=1 \cdots n
$$


Where $\gamma_{i}^{\varepsilon}$ is the MVC-ID of the error vector $\varepsilon$. Thus, the Equation 15 can be written as:

$$
p=\sum_{i=1}^{n}\left(\gamma_{i}+\gamma_{i}^{\varepsilon}\right) v_{i}+\varepsilon, \quad i=1 \cdots n
$$

- If, in the Equation 17, the error $\|\varepsilon\|>10^{-5}$, we repete the above step, otherwise the process finish and we have:

$$
\mu_{i}=\gamma_{i}+\gamma_{i}^{1}+\gamma_{i}^{2}+\cdots+\gamma_{i}^{k}, i=1 \cdots n
$$

the MVC-ID of the point $p$. Where $k$ is the number of interaction necessary.

The influence of a control vertex on a region of the model is inversely proportional to its distance from it. Although our approach does not completely eliminate the negative influence of a control vertice, by replacing the Euclidean distance $r_{i}$ by a interior distance $\hat{r}_{i}$ taken inside the cage control, we increase the distance of a given control vertex to regions of the model not related to it, consequently, diminishing its influence on this regions.

One potential disadvantage of MVC-ID compared to the original Mean Value Coordinates is the computational cost of the coordinates derivation. The interactive method, applied to solve the reconstruction problem, increases $\mathrm{k}$ times the computational cost of the coordinates derivation, where $\mathrm{k}$ is the number of interaction necessary. However, as the coordinates are computed only once and stored, the cost of evaluating the deformations with the MVC-ID is identical to that of deformations based on the original Mean Value Coordinates.

\section{Results and Comparisons}

Replacing the Euclidean distance by an interior distance during the derivation of the coordinates results in a significant reduction of the influence of a control vertex on regions of the model not related to it. In the Figure 7 we highlight the regions of greatest influence of the orange control vertex on the model with the original MVC and our coordinates. The red region indicates a positive influence and the green region the negative influence.

The Table 1 presents the comparison between the coordinates of the point in blue on the model, related to the orange control vertex, using both original MVC and MVC-ID. In both case, the reduction of the negative influence is greater than $73 \%$.

Table 1. Influence of selected points in Figure 7.

\begin{tabular}{||c||c||c||}
\hline Method & Figure (a) & Figure (b) \\
\hline \hline MVC & -0.26326 & -0.12624 \\
\hline \hline MVC-ID & -0.07039 & -0.02052 \\
\hline \hline Reduction & $73.26 \%$ & $83.74 \%$ \\
\hline \hline
\end{tabular}

In the Figures 9 and 8 we compare the deformation obtained by the original MVC, the Harmonic coordinates and

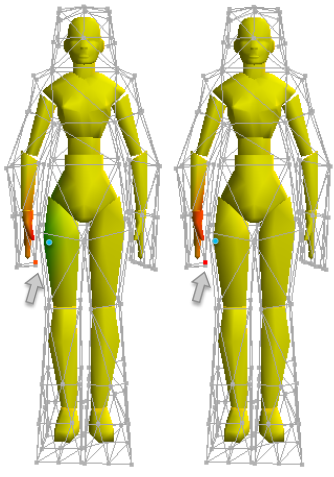

(a)

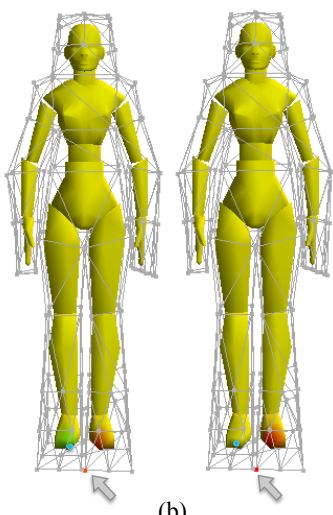

(b)
Figure 7. (a) Comparison of the effect of the control vertex highlighted in the hand between original MVC, on the left, and our MVC-ID, on the right. (b) Analogous comparison for a control vertex of the foot.

our coordinates. For a comparison of the global behavior of both deformation methods, we opt for to analyze the change in model volume and surface area before and after the undergoing deformation. The Tables 2 and 3 indicate that, even without changing the position of the cage control vertex, the surface area of the model obtained by the deformation with $\mathrm{HC}$ is the one that undergoes the greatest change. This occur because the solution of Laplace's equation, which is used to construct the coordinates, is initially computed only on the vertices of the grid. Thus, the linear precision of the method is guaranteed only on cells of this grid. The value of the function on points of the model is given by trilinear interpolation, which makes the deformation results directly dependent of the grid density. The results obtained using our coordinates yield a non-significant reduction of $0.2 \%$ in surface area.

Table 2. Surface area of deformations obtained in Figure 8.

\begin{tabular}{||c||c||}
\hline \hline Method & Surface area \\
\hline \hline Original model & 347.176 \\
\hline \hline Mean Value Coord. & 347.176 \\
\hline \hline Mean Value Coord. -ID & 346.448 \\
\hline \hline Harmonic Coord. & 360.053 \\
\hline \hline
\end{tabular}

Table 3. Volume and surface area of deformations shown in Figure 9.

\begin{tabular}{||c||c||c||}
\hline \hline Method & Surface area & Volume \\
\hline \hline Original Model & 243.349 & 248.004 \\
\hline \hline MVC & 239.913 & 243.172 \\
\hline \hline MVC-ID & 242.102 & 246.523 \\
\hline \hline HC & 254.355 & 259.433 \\
\hline \hline
\end{tabular}

It is possible to observe also in the Figure 9(b), where we present a deformation process using the original Mean Value coordinates, the undesired deformation on the model's foot. 

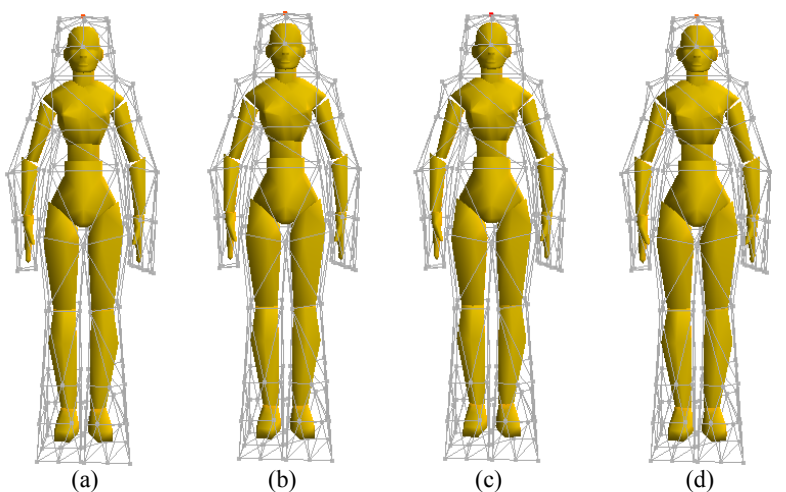

Figure 8. (a) Original model. (b) Result obtained using MVC. (c) Result obtained using MVC-ID. (d) Result obtained with HC.

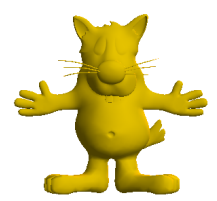

(a)

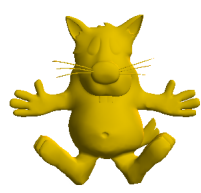

(b)

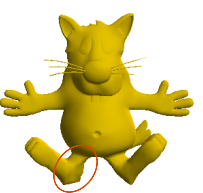

(c)

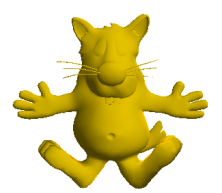

(d)
Figure 9. (a) Original Model. (b) Deformation generated by the original MVC. The red circle indicates the consequences of the strong negative influence of some control vertices on the model. (c) Deformation generated by MVC-ID. (d) Deformation generated by Harmonic Coordinates.

In the Figure 9(c) we perform the same deformation, now using our coordinates.

The Figure 8 illustrates the female robot model deformation obtained using each method, with the cage control vertices in their original positions. We opt for to present first the result obtained by the recovering of the model with the cage control vertices in their original position.

The Figures 10 and 11 illustrate the deformations of the arms and legs of the female robot model and compare the results obtained using both our coordinates and the original MVC. Tables 4 and 5 present the numerical results obtained from the analyze of the variation in the female robot model's volume and surface area. In both cases it is possible to observe that the deformations using MVC-ID yield better results in terms of the volume and surface area of the model.

Table 4. Volume and surface area of deformations shown in Figure 10.

\begin{tabular}{||c||c||c||c||c||}
\hline \hline Method & Surface area & $A_{f} / A_{i}$ & Volume & $V_{f} / V_{i}$ \\
\hline \hline Original Model & 347.176 & - & 231.149 & - \\
\hline \hline MVC & 331.279 & 0.954 & 210.492 & 0.910 \\
\hline \hline MVC-ID & 342.702 & 0.9987 & 226.821 & 0.981 \\
\hline \hline
\end{tabular}

As observed in Figure 12 and Table 6, in cases where the negative coordinates of the original method do not lead to undesirable changes, the deformations given by our coordinates
Table 5. Volume and surface area of deformations shown in Figure 11.

\begin{tabular}{||c||c||c||c||c||}
\hline \hline Method & Surface area & $A_{f} / A_{i}$ & Volume & $V_{f} / V_{i}$ \\
\hline \hline Original Model & 347.176 & - & 231.149 & - \\
\hline \hline MVC & 336.158 & 0.968 & 217.562 & 0.941 \\
\hline \hline MVC-ID & 340.207 & 0.979 & 223.813 & 0.968 \\
\hline \hline
\end{tabular}

Table 6. Volume and surface area of deformations shown in Figure 12.

\begin{tabular}{||c||c||c||}
\hline \hline Method & Surface area & Volume \\
\hline \hline Original Model & 219.915 & 273.465 \\
\hline \hline MVC & 257.953 & 334.390 \\
\hline \hline MVC-ID & 259.623 & 336.109 \\
\hline \hline
\end{tabular}

display the same behavior as those deformations obtained using MVC.
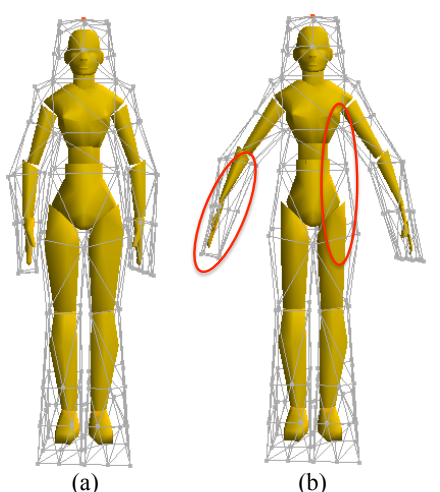

(b)

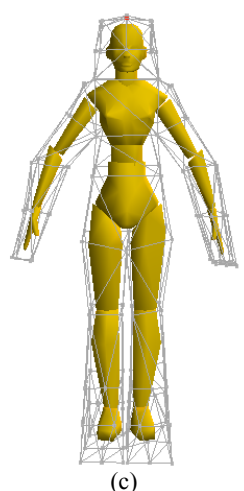

(c)
Figure 10. (a) Original Model. (b) Deformation obtained using original MVC. The red circles highlight the consequences of the strong negative influence of some control vertices on the model. (a) Deformation generated by MVC-ID.

\section{Conclusions}

In this work we propose a modification on the derivation of MVC, the Euclidean distance used in the original derivation is replaced by a distance taken inside the cage control, which respect the shape of the model and cage control. The use of the interior distance is effective in reducing the influence of control points in regions not related to them. Consequently, the deformations generated using our coordinates do not present the undesirable behavior observed in deformations generated using the original MVC. For future work we should include the investigation of the use of MVC-ID in the application of volumetric textures, as suggested by Ju et al. [5], and compare it with the results obtained with the MVC. 


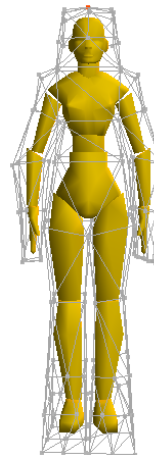

(a)

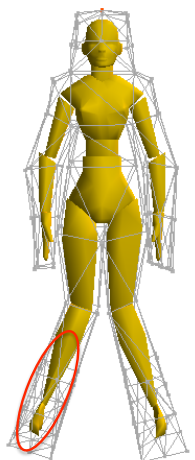

(b)

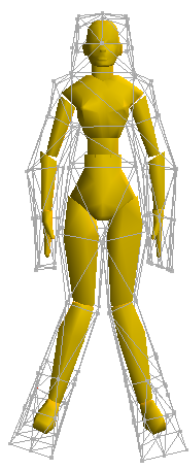

(c)
Figure 11. (a) Original Model. (b) Deformation obtained using original MVC. The red circle indicates the consequences of the strong negative influence of some control vertices on the model. (c) Deformation generated by MVC-ID.
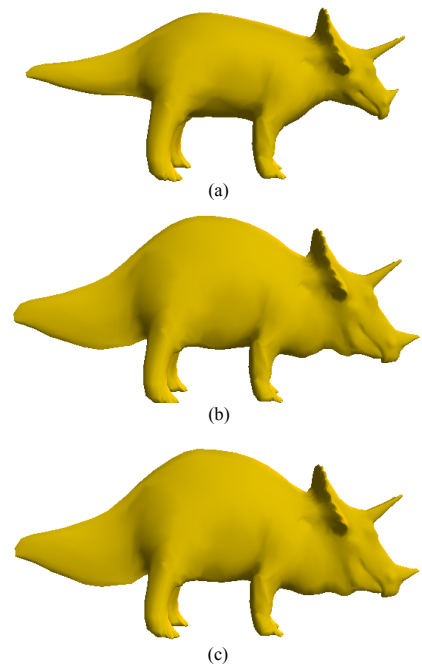

Figure 12. (a) Original Model. (b) Deformation generated by the original MVC. (c) Deformation generated by MVC-ID.

\section{Author contributions}

This work is a continuation of the Lis Custdio's master's thesis, under Sinesio Pesco guidance.

\section{References}

[1] SEDERBERG, T. W.; PARRY, S. R. Free-form deformation of solid geometric models. SIGGRAPH Comput. Graph., v. 20, n. 4, p. 151-160, 1986.

[2] COQUILLART, S. Extended free-form deformation: a sculpturing tool for $3 \mathrm{~d}$ geometric modeling. SIGGRAPH Comput. Graph., v. 24, n. 4, p. 187-196, 1990.
[3] KOBAYASHI, K. G.; OOTSUBO, K. t-ffd: free-form deformation by using triangular mesh. In: TURKIYYAH, G.; BRUNET, P. (Ed.). Proceedings of the eighth ACM symposium on Solid modeling and applications. Seattle, WA, USA: ACM, 2003. (SM '03, v. 1).

[4] FLOATER, M. S. Mean value coordinates. Comput Aided Geom Des, v. 20, n. 1, p. 19-27, 2003.

[5] JU, T.; SCHAEFER, S.; WARREN, J. Mean value coordinates for closed triangular meshes. ACM Trans Graph., v. 24, n. 3, p. 561-566, 2005.

[6] FLOATER, M. S.; KOS, G.; REIMERS, M. Mean value coordinates in 3d. Comput Aided Geom Des, v. 22, n. 7, p. 623-631, 2005.

[7] LI, X.-Y.; JU, T.; HU, S.-M. Cubic mean value coordinates. ACM Trans Graph., v. 32, n. 4, p. 126:1-10, 2013.

[8] RUSTAMOV, R. M. Barycentric coordinates on surfaces. Comput. Graphs. Forum, v. 29, n. 5, p. 1507-1516, 2010.

[9] FARBMAN, Z. et al. Coordinates for instant image cloning. ACM Trans. Graph., v. 28, n. 3, p. 67:1-9, 2009.

[10] JOSHI, P. et al. Harmonic coordinates for character articulation. ACM Trans Graph., v. 26, n. 3, p. 71:1-71:10, 2007.

[11] LIPMAN, Y. et al. Gpu-assisted positive mean value coordinates for mesh deformations. In: SGP '07. Proceedings of the Fifth Eurographics Symposium on Geometry Processing. Aire-la-Ville, Switzerland: Eurographics Association, 2007. v. 1, p. 117-123.

[12] LIPMAN, Y.; LEVIN, D.; COHEN-OR, D. Green coordinates. ACM Trans. Graph., v. 27, n. 3, p. 78:1-78:10, 2008.

[13] SCHAEFER, J. M. andScott. Moving least squares coordinates. Comput. Graphs. Forum, v. 29, n. 5, p. 1517-1524, 2010.

[14] CUSTODIO, L. Extensões de Coordenadas Baricêntricas para Deformação de Malhas. Dissertação (Mestrado) - Departamento de Matemática, PUC-Rio, Rio de janeiro, Brazil, 2010.

[15] RUSTAMOV, R. M.; LIPMAN, Y.; FUNKHOUSER, T. Interior distance using barycentric coordinates. In: Proceedings of the Symposium on Geometry Processing. Aire-la-Ville, Switzerland: Eurographics Association, 2009. (SGP '09, v. 1), p. 1279-1288.

[16] JU, T.; LIEPA, P.; WARREN, J. A general geometric construction of coordinates in a convex simplicial polytope. Comput Aided Geom Des, v. 24, n. 3, p. 161-178, 2007. 